AUTORES:

Celina Aparecida G Lima

Maria de Fatima de Matos Maia

Berenilde Valeria de Sousa ${ }^{1}$

Thatiana Maia Tolentino ${ }^{2}$

Maria Fernanda S F Brito

Lucineia de Pinho ${ }^{1}$

Marise Fagundes Silveira

${ }^{1}$ Universidade Estadual de Montes

Claros (UNIMONTES). Montes Claros,

Minas Gerais (Brasil)

${ }^{2}$ Faculdades Santo Agostinho,

Sete Lagoas, Minas Gerais (Brasil)

\section{Comportamento de risco}

\section{relacionado à segurança}

\section{universitários do norte}

\section{de Minas Gerais}

\section{PALAVRAS CHAVE:}

Saúde. Segurança. pessoal em jovens

Comportamento de risco.

Risk behavior related to personal safety in college young students of the north

of Minas Gerais

\section{ABSTRACT}

This study aimed to estimate the prevalence of risk behaviors related to personal safety, as well to identify associated factors. This is a cross-sectional study with 902 academic students. We used the instrument Youth Risk Behavior Survey - College. Prevalences were estimated at intervals of $95 \%$ of confindence and reasons of crude and adjusted prevalences by Poisson regression. Most of the students was female (67.1\%) and aged up to 21 years $(56.2 \%)$. Risk behaviors most prevalent: Not using seat belts (34.7\%) and alcohol associated with the vehicle direction (39.6\%). Main associations: men and involvement in fights $(P R=2.04)$, age up to 21 years and non-use of seat belts $(P R=1.32)$ and no helmet $(P R=1.87)$; area of exact sciences and drink associated with the direction ( $P R=0.64)$; humanities and drink associated with the direction ( $P R=0.73)$; area of applied social sciences and drink associated with the direction $(P R=0.76)$; better education of the mother and drink associated with the direction $(P R=1.43)$; economic classes $C$ and $D$ and drink associated with the direction $(P R=0.77)$. Men have more chances of involvement in fights and, for younger, of not using the safety belt and helmet.

KEY-WORDS:

Risk behavior. Health. Safety. 
A saúde está associada ao estilo de vida de cada pessoa e aos seus padrões de comportamento. Atualmente, devido aos hábitos adotados, os jovens estão cada vez mais vulneráveis a situações que colocam em risco a sua saúde (Barbosa, 2014).

Dentre os comportamentos de risco para a saúde (CRS), os relacionados à segurança pessoal, como o não uso de cinto de segurança e capacete, o consumo de bebida alcoólica associada à direção de veículo e o envolvimento em brigas, têm sido considerados importantes na ocorrência de lesões corporais e no aumento de mortes no trânsito, especialmente entre os jovens, o que levam a altos custos emocionais, sociais e gastos com assistência à saúde (Neto et al., 2010).

Devido ao aumento do número de veículos e mudanças no estilo de vida das pessoas, os acidentes de trânsito tem se tornado um grave problema social, configurando-se como causas de mortalidade e morbidade da população em geral (Kanchan, Kulkarni, Bakkannavar, Kumar, \& Unnikrishnan, 2012). Nos Estados Unidos, em 2014, 71\% de todas as mortes entre pessoas com idades entre 10-24 anos resultaram de quatro causas: acidentes com veículos automotores (23\%), outras lesões não intencionais (17\%), homicídio (14\%) e suicídio (17\%) (1) (Center for Disease Control [CDC], 2015). Segundo a Organização das Nações Unidas (ONU), acidentes no trânsito matam cerca de 1,25 milhões de pessoas por ano, e é a principal causa de morte entre os jovens com idades entre 15 e 29 anos.

No cenário nacional, de acordo com a Pesquisa Nacional de Saúde (PNS, 2013), 20.6\% das pessoas de 18 anos ou mais não usavam cinto de segurança no banco da frente quando andavam de automóvel, $19.9 \%$ não usavam capacete, como passageiro e $16.6 \%$ quando dirigiam uma motocicleta, e a proporção de indivíduos que conduziram veículo motorizado, carro ou motocicleta, após o consumo de bebida alcoólica foi de 24.3\%. Em 2013, o valor gasto pelo Sistema Único de Saúde (SUS), com internações de acidentes com motocicletas foi de R\$ 112 milhões (Ministério da Saúde, 2015).

Apesar da magnitude desse problema, estudos sobre comportamentos de risco na população jovem no Brasil e os fatores associados ainda tem sido pouco investigados tanto na literatura nacional e internacional. Tal conhecimento se torna imprescindível para subsidiar políticas públicas de prevenção dos agravos oriundos dos comportamentos de risco. Nesse sentido o objetivo do presente estudo foi estimar a prevalência de comportamento de risco à saúde relacionado à segurança pessoal, em uma população de universitários no norte do estado de Minas Gerais e identificar os seus fatores associados.
O presente estudo é um recorte do projeto "Comportamentos de risco à saúde dos universitários no norte do estado de Minas Gerais", realizado pelo Grupo Integrado de Pesquisa em Psicologia do Esporte, Exercício e Saúde, Saúde Ocupacional e Mídia(GIPESOM), vinculado à Universidade Estadual de Montes Claros - Unimontes. Trata-se de um estudo transversal, cuja população-alvo foi constituída por 12015 estudantes matriculados nos cursos presenciais da Unimontes, em 2013. A Universidade Estadual de Montes Claros - Unimontes é uma instituição pública de ensino superior, localizada na cidade de Montes Claros, MG, centro convergente e polarizador dos demais municípios da região do Norte de Minas Gerais. Ela oferece formação nas áreas de saúde, educação, ciências sociais aplicadas, ciências humanas e ciências exatas e tecnológicas, no campus-sede de Montes Claros, com 158 cursos, bem como nos outros campi: Almenara, Bocaiúva, Brasília de Minas, Espinosa, Janaúba, Januária, Joaíma, Paracatu, Pirapora, Salinas, São Francisco e Unaí.

AMOSTRA

O processo de amostragem foi probabilístico por conglomerado em dois estágios. No $1^{0}$ estágio, por amostragem aleatória simples (AAS), foram selecionados $50 \%$ dos cursos de cada área de conhecimento da instituição (Ciências Sociais Aplicadas, Ciências Exatas e Tecnológicas, Ciências Humanas e Ciências Biológicas e da Saúde); no 2oestágio, por AAS, sorteou-se $25 \%$ das turmas em cada curso previamente selecionado. Todos os alunos dessas turmas presentes no momento da aplicação do questionário foram convidados a participar da pesquisa. Para o cálculo do tamanho da amostra foram considerados os seguintes parâmetros: prevalência de $50 \%$ para o comportamento de risco, nível de confiança de $95 \%$ e erro de $5 \%$. Foi também realizada a correção pelo efeito do desenho adotando-se deff igual a 2 e acréscimo de $20 \%$ para taxa de não-resposta, compreendendo, assim, uma amostra de 960 indivíduos.

INSTRUMENTO

Aplicou-se o instrumento Youth Risk Behavior Survey - College (YRBS-C), traduzido e adaptado transculturalmente para o português (Guedes \& Lopes, 2010). Esse instrumento contém 78 questões relacionadas aos CRS: segurança pessoal (10 itens), violência, (cinco itens), suicídio (quatro itens), uso de tabaco (oito itens), consumo de bebidas alcoólicas (três itens), uso de maconha (três itens), uso de outras drogas (10 itens), atividade sexual (15 itens), peso corporal (oito itens), alimentação (sete itens) e atividade física (cinco itens) (Bernadelli Junior, 2010). 
PROCEDIMENTOS

No presente estudo foram investigados quatro comportamentos de risco relacionados à segurança pessoal: não uso de cinto de segurança, não uso de capacete, bebida alcoólica associada à direção de veículo e envolvimento em brigas, bem como variáveis relacionadas às características demográficas, acadêmicas e socioeconômicas. Utilizou-se o Critério de Classificação Econômica Brasil (CCEB) da Associação Brasileira de Empresas de Pesquisa (ABEP, 2013) para identificar a classe econômica dos universitários (QUADRO 1)

QUADRO 1. Variáveis do estudo, medidas utilizadas e categorias adotadas na análtse dos dados da amostra de universitários no norte de Minas Gerais, Brasil, 2013.

\begin{tabular}{|c|c|c|}
\hline VARIÁVEIS & MEDIDAS (AUTORREFERIDAS) & CATEGORIAS \\
\hline \multicolumn{3}{|l|}{$\begin{array}{l}\text { COMPORTAMENTOS } \\
\text { DE RISCO }\end{array}$} \\
\hline Não uso de cinto segurança & $\begin{array}{l}\text { Frequência do uso de cinto de segurança } \\
\text { quando estava dirigindo ou quando estava } \\
\text { em um carro dirigido por outra pessoa. }\end{array}$ & $\begin{array}{l}\text { Sem risco: sempre } \\
\text { Com risco: nunca/raramente/ } \\
\text { algumas vezes/ na maioria das } \\
\text { vezes }\end{array}$ \\
\hline Não uso de capacete & $\begin{array}{l}\text { Frequência do uso de capacete quando andou } \\
\text { de motocicleta nos } 12 \text { meses que antecederam } \\
\text { a coleta dos dados. }\end{array}$ & $\begin{array}{l}\text { Sem risco: sempre/ não andou } \\
\text { Com risco: nunca/raramente/ } \\
\text { algumas vezes/ na maioria } \\
\text { das vezes }\end{array}$ \\
\hline $\begin{array}{l}\text { Bebida alcoólica associada à } \\
\text { direção de veículo }\end{array}$ & $\begin{array}{l}\text { Frequência de utilizacacão de veículo dirigido pela } \\
\text { própria pessoa ou outra, quando ingeriu bebida } \\
\text { alcoólica, nos } 30 \text { dias que antecederam a coleta } \\
\text { dos dados. }\end{array}$ & $\begin{array}{l}\text { Sem risco: nenhuma vez } \\
\text { Com risco: uma ou mais vezes }\end{array}$ \\
\hline Envolvimento em brigas & $\begin{array}{l}\text { Frequência com que se envolveu em agressões } \\
\text { físicas nos } 12 \text { meses que antecederam a coleta } \\
\text { dos dados. }\end{array}$ & $\begin{array}{l}\text { Sem risco: nenhuma vez } \\
\text { Com risco: uma ou mais vezes }\end{array}$ \\
\hline \multicolumn{3}{|c|}{ CARACTERÍSTICAS DEMOGRÁFICAS E SOCIOECONÔMICAS } \\
\hline Gênero & Classificação dos acadêmicos quanto ao gênero & $\begin{array}{l}\text { Feminino } \\
\text { Masculino }\end{array}$ \\
\hline Faixa etária & Faixa de idade do acadêmico & $\begin{array}{l}\text { Acima de } 21 \text { anos } \\
\text { Até } 21 \text { anos }\end{array}$ \\
\hline Cor de pele & Cor de pele autorrelatada & $\begin{array}{l}\text { Branca } \\
\text { Outras: negro/asiático /índio }\end{array}$ \\
\hline Estado Civil & Estado civil do acadêmico & $\begin{array}{l}\text { Com companheiro: casado(a)/ } \\
\text { união estável } \\
\text { Sem companheiro: solteiro/ } \\
\text { viúvo/divorciado/ Separado }\end{array}$ \\
\hline Trabalho & Condição trabalhista & $\begin{array}{l}\text { Trabalha com vínculo } \\
\text { Trabalha sem vínculo } \\
\text { Não trabalha }\end{array}$ \\
\hline Escolaridade paterna & Escolaridade do pai & $\begin{array}{l}<4 \text { anos } \\
4 \text { a } 10 \text { anos } \\
11 \text { anos }\end{array}$ \\
\hline Escolaridade materna & Escolaridade da mãe & $\begin{array}{l}<4 \text { anos } \\
4 \text { a } 10 \text { anos } \\
11 \text { anos }\end{array}$ \\
\hline Classe econômica & $\begin{array}{l}\text { Segundo Critério de Classificação } \\
\text { Econômica Brasil (CCEB) }\end{array}$ & $\begin{array}{l}\text { Classe A e B } \\
\text { Classe C e D }\end{array}$ \\
\hline
\end{tabular}

\begin{tabular}{lll}
\hline & \multicolumn{1}{c}{ CARACTERÍSTICAS ACADÊMICAS } & \\
\hline Área de Graduação & $\begin{array}{l}\text { Área em que estava cursando a graduação } \\
\text { no período da coleta de dados }\end{array}$ & $\begin{array}{l}\text { Ciências Biológicas e da Saúde } \\
\text { Ciências Humanas } \\
\text { Ciências Exatas e Tecnológicas } \\
\text { Ciênncias Sociais Aplicadas }\end{array}$ \\
\hline Campus & $\begin{array}{l}\text { Campus em que estava cursando a graduação } \\
\text { no período da coleta de dados }\end{array}$ & $\begin{array}{l}\text { Montes Claros } \\
\text { Outros }\end{array}$ \\
\hline Turno de estudo & $\begin{array}{l}\text { Turno do dia em que estava matriculado } \\
\text { no perído da coleta de dados }\end{array}$ & $\begin{array}{l}\text { Diurno } \\
\text { Noturno }\end{array}$ \\
\hline Período de estudo & Período em que estava cursando & $\begin{array}{l}\text { 50 período em diante } \\
1^{0} \text { ao 40 período }\end{array}$ \\
\hline
\end{tabular}

Previamente à aplicação dos questionários, os estudantes foram informados sobre os objetivos da pesquisa e sobre a preservação do anonimato. Os questionários foram aplicados em cada sala de aula, selecionada por equipe treinada, sob a supervisão dos pesquisadores responsáveis pelo projeto. Após o preenchimento, os estudantes depositaram os questionários em um envelope sem identificação.

ANÁLISE ESTATÍSTICA

Para descrever os acadêmicos quanto às variáveis analisadas foram utilizadas frequências, simples e relativas. Foram também estimados intervalos de $95 \%$ de confiança para as prevalências dos comportamentos de risco relacionados à segurança pessoal. Os quatro comportamentos de risco investigados foram considerados variáveis dependentes desse estudo e as características demográficas, acadêmicas e socioeconômicas constituíram as variáveis independentes. Para avaliar a magnitude da associação entre as variáveis dependentes e as variáveis independentes foram estimadas razões de prevalências (RP) brutas e ajustadas, por meio de modelos de regressão de Poisson, com variância robusta. Inicialmente foram realizadas análises bivariadas (teste do qui-quadrado) e as variáveis que apresentaram nível descritivo (valor-p) até.20 foram selecionadas para o modelo múltiplo, cujo nível de significância adotado foi de.05. 0 teste deviance foi adotado para avaliar a qualidade do modelo ajustado. Todas as análises estatísticas foram realizadas utilizando-se o programa Predictive Analytics Software (PASW) ${ }^{\circledR}$ versão 19.0 para Windows ${ }^{\circledR}$.

Esse estudo foi aprovado pelo Comitê de Ética em Pesquisa da instituição, (Parecer no 30679/2012), e todos os universitários que concordaram em participar do estudo assinaram um Termo de Consentimento Livre e Esclarecido - TCLE.

\section{RESULTADOS}

0 questionário foi respondido por 902 acadêmicos, obtendo-se uma taxa de resposta de $94 \%$, dos quais $67.1 \%$ eram do gênero feminino e $56.2 \%$ tinham idade até 21 anos. As prevalências dos comportamentos de risco relacionados à segurança pessoal foram: não uso 
de cinto de segurança (34.7\%), não uso de capacete (5.3\%), bebida alcoólica associada à direção de veículo (39.6\%) e envolvimento em brigas (4.2\%) (QUADRO 2). Mais da metade dos jovens (57.4\%) apresentaram simultaneamente os comportamentos de risco mais prevalentes (FIGURA 1 ).

QUADRO 2. Distribuição dos universitários segundo características demográficas, socioeconômicas, acadêmicas e comportamentos de risco à saúde relacionados à segurança pessoal, norte de Minas Gerais, Brasil. 2013.

\begin{tabular}{|c|c|c|c|}
\hline VARIÁVEL & CATEGORIA & $\mathrm{N}$ & $\%$ \\
\hline \multicolumn{4}{|c|}{ CARACTERÍSTICAS DEMOGRÁFICAS E SOCIOECONÔMICAS } \\
\hline \multirow[t]{2}{*}{ Gênero } & Feminino & 605 & 67.1 \\
\hline & Masculino & 297 & 32.9 \\
\hline \multirow[t]{2}{*}{ Faixa Etária } & Acima de 21 anos & 395 & 43.8 \\
\hline & Até 21 anos & 507 & 56.2 \\
\hline \multirow[t]{2}{*}{ Cor de pele } & Branca & 343 & 38.0 \\
\hline & Outra & 559 & 62.0 \\
\hline \multirow[t]{2}{*}{ Estado Civil } & Com companheiro & 121 & 13.4 \\
\hline & Sem companheiro & 781 & 86.6 \\
\hline \multirow[t]{3}{*}{ Trabalho } & Trabalha com vínculo & 414 & 45.9 \\
\hline & Trabalha sem vínculo & 113 & 12.5 \\
\hline & Não trabalha & 375 & 41.6 \\
\hline \multirow[t]{3}{*}{ Escolaridade paterna } & $<4$ anos & 253 & 28.0 \\
\hline & 4 a 10 anos & 384 & 42.6 \\
\hline & 11 anos & 265 & 29.4 \\
\hline \multirow[t]{3}{*}{ Escolaridade materna } & $<4$ anos & 168 & 18.6 \\
\hline & $4 \mathrm{a} 10$ anos & 343 & 38.0 \\
\hline & 11 anos & 391 & 43.3 \\
\hline \multirow[t]{2}{*}{ Classe econômica } & Classe A e B & 161 & 17.8 \\
\hline & Classe C e D & 740 & 82.2 \\
\hline \multicolumn{4}{|c|}{ CARACTERÍSTICAS ACADÊMICAS } \\
\hline \multirow[t]{4}{*}{ Área de graduação } & Ciências Biológicas e da Saúde & 184 & 20.4 \\
\hline & Ciências Humanas & 327 & 36.2 \\
\hline & Ciências Exatas e Tecnológicas & 144 & 16.0 \\
\hline & Ciências Sociais Aplicadas & 247 & 27.4 \\
\hline \multirow[t]{2}{*}{ Campus } & Montes Claros & 596 & 66.1 \\
\hline & Outros & 306 & 33.9 \\
\hline \multirow[t]{2}{*}{ Turno de estudo } & Diurno & 378 & 41.9 \\
\hline & Noturno & 524 & 58.1 \\
\hline \multirow[t]{2}{*}{ Período de graduação } & 1ํao 4ํp período & 532 & 59.0 \\
\hline & 5o período ou mais & 370 & 41.0 \\
\hline
\end{tabular}

\begin{tabular}{llcc}
\hline COMPORTAMENTOS DE RISCO & & $\mathrm{N}$ & $\%\left(\mathrm{IC}_{95 \%}\right)$ \\
\hline Não uso de cinto segurança & Sem risco & 589 & $65.3(62.2-68.4)$ \\
\hline & Com risco & 313 & $34.7(31.6-37.8)$ \\
\hline Não uso de capacete & Sem risco & 854 & $94.7(93.2-96.2)$ \\
\hline & Com risco & 048 & $5.3(3.8-6.8)$ \\
\hline $\begin{array}{l}\text { Bebida alcoólica associada } \\
\text { a direção de veículo }\end{array}$ & Sem risco & 545 & $60.4(57.2-63.6)$ \\
\hline & Com risco & 357 & $39.6(36.4-42.8)$ \\
\hline Envolvimento em brigas & Sem risco & 864 & $95.8(94.5-97.1)$ \\
\hline & Com risco & 038 & $4.2(2.9-5.5)$ \\
\hline
\end{tabular}

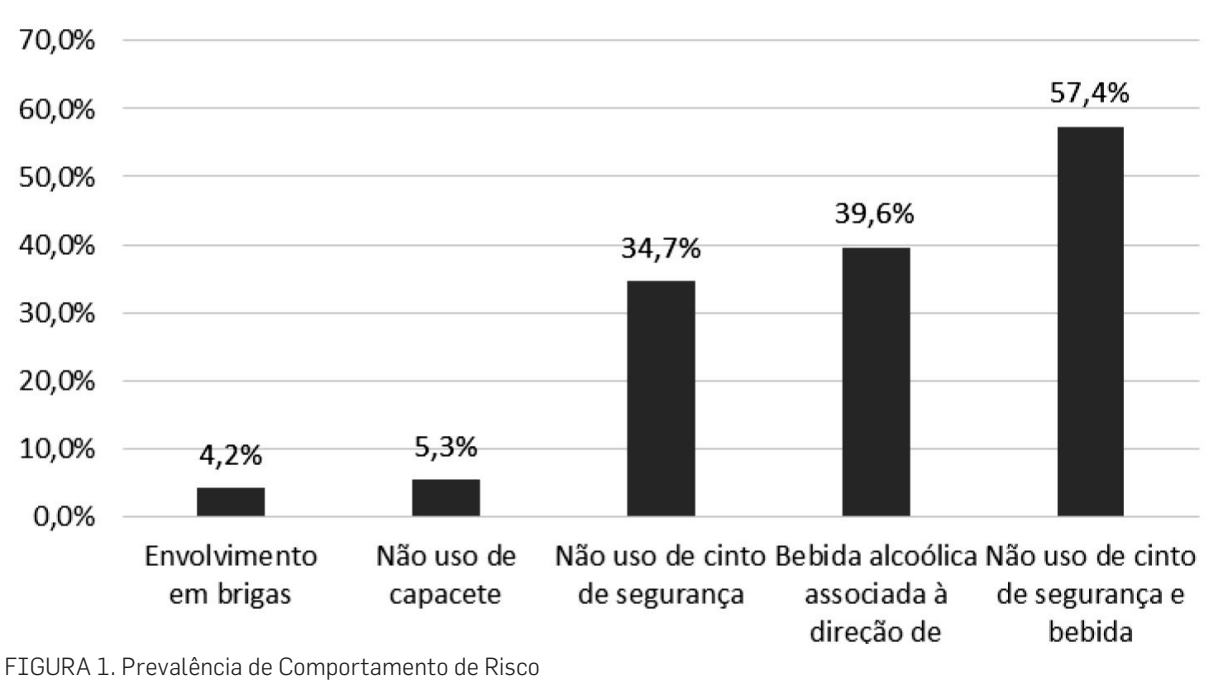

As prevalências dos comportamentos de risco à saúde relacionados à segurança pessoal (CRSRSP), segundo características demográficas socioeconômicas e acadêmicas dos estudantes estão apresentadas no quadro 3. Os universitários do gênero masculino apresentaram prevalências maiores em quase todos os CRSRSP. Já os mais jovens apresentaram maiores prevalências em todos os comportamentos de risco analisados. Os acadêmicos que autodeclararam de cor branca apresentaram maior prevalência do uso de bebida alcoólica associada à direção de veículo (44.3\%).

Os estudantes da área de biológicas e da saúde mostraram uma prevalência maior de bebida alcoólica associada à direção de veículo $(50.0 \%)$. Os acadêmicos que realizaram trabalho com vínculo apresentaram maior prevalência de bebida alcoólica associada à direção de veículo (45.4\%). Aqueles cuja escolaridade do pai e da mãe era mais alta apresentaram maiores prevalências do não uso de cinto de segurança e bebida associada à direção de veículo. 0 não uso de capacete foi mais prevalente entre os jovens cuja escolaridade 
paterna era mais baixa (8.3\%). Os acadêmicos de classe econômica A e B apresentaram maior prevalência do não uso do cinto de segurança (40.4\%) e de bebida alcoólica associada à direção de veículo (53.4\%) (QUADRO 3).

QUADRO 3. Prevalência de comportamentos de risco para a saúde relacionados

à segurança pessoal segundo características demográficas socioeconômicas e acadêmicas de universitários no norte de Minas Gerais, Brasil, 2013.

\begin{tabular}{|c|c|c|c|c|}
\hline & $\begin{array}{l}\text { NÃO USO DE CINTTO } \\
\text { DE SEGURANÇA }\end{array}$ & $\begin{array}{c}\text { NÃO USO } \\
\text { DE CAPACETE }\end{array}$ & $\begin{array}{l}\text { BEBIDA ALCOÓLICA } \\
\text { ASSOCIADA } \\
\text { A DIREÇÃO } \\
\text { DE VEÍCULO }\end{array}$ & $\begin{array}{l}\text { ENVOLVIMENTO } \\
\text { EM BRIGAS }\end{array}$ \\
\hline & $\%$ & $\%$ & $\%$ & $\%$ \\
\hline \multicolumn{5}{|l|}{ GÊNERO } \\
\hline Feminino * & 34.0 & 5.5 & 37.9 & 3.1 \\
\hline Masculino & 36.0 & 5.1 & 43.1 & 6.4 \\
\hline RP (IC 95\%) & $1.06(.88-1.28)$ & $.93(0.51-1.68)$ & $1.14(.97-1.34)$ & $2.04(1.10-3.79)$ \\
\hline \multicolumn{5}{|l|}{ FAIXA ETÁRIA } \\
\hline Acima de 21 anos * & 30.1 & 4.1 & 37.5 & 2.8 \\
\hline Até 21 anos & 38.3 & 6.3 & 41.2 & 5.3 \\
\hline RP (IC 95\%) & $1.27(1.05-1.53)$ & $1.56(.87-2.80)$ & $1.10(.93-1.30)$ & $1.91(.96-3.81)$ \\
\hline \multicolumn{5}{|l|}{ ÁREA DA GRADUAÇÃo } \\
\hline Biológicas e da Saúde * & 39.7 & 4.3 & 50.0 & 3.3 \\
\hline Humanas & 39.1 & 6.1 & 35.5 & 3.4 \\
\hline Exatas e Tecnológicas & 25.0 & 7.6 & 30.6 & 4.9 \\
\hline Sociais Aplicadas & 30.8 & 3.6 & 42.5 & 5.7 \\
\hline \multirow[t]{3}{*}{ RP (IC 95\%) } & $.99(.79-1.23)$ & $1.41(.63-3.13)$ & $.71(.58-.87)$ & $1.03(.39-2.74)$ \\
\hline & $.63(.45-.88)$ & $1.76(.73-4.25)$ & $.61(.46-81)$ & $1.49(.51-4.34)$ \\
\hline & $.78(.60-1.00)$ & $.84(.33-2.13)$ & $.85(.69-1.04)$ & $1.74(.68-4.44)$ \\
\hline \multicolumn{5}{|l|}{ TRABALHO } \\
\hline Trabalha com vínculo* & 36.0 & 5.1 & 45.4 & 5.1 \\
\hline Trabalha sem vínculo & 38.9 & 7.1 & 34.5 & .9 \\
\hline Não trabalha & 32.0 & 5.1 & 34.7 & 4.3 \\
\hline \multirow[t]{2}{*}{ RP (IC 95\%) } & $1.08(.83-1.41)$ & $1.40(.64-3.07)$ & $.76(.58-1.00)$ & $.17(.02-1.28)$ \\
\hline & $.89(.73-1.08)$ & $1.00(.55-1.83)$ & $.76(.64-.91)$ & $.84(.45-1.59)$ \\
\hline \multicolumn{5}{|l|}{ ESCOLARIDADE PAI } \\
\hline$<4$ anos ${ }^{*}$ & 3.2 & 8.3 & 32.8 & 3.6 \\
\hline $4 \mathrm{a} 10$ anos & 34.1 & 5.5 & 39.1 & 4.2 \\
\hline 11 anos & 37.0 & 2.3 & 46.8 & 4.9 \\
\hline \multirow[t]{2}{*}{ RP (IC 95\%) } & $1.03(.82-1.29)$ & $.66(.37-1.18)$ & $1.19(.96-1.48)$ & $1.17(.53-2.61)$ \\
\hline & $1.11(.88-1.41)$ & $.27(.11-0.67)$ & $1.43(1.15-1.77)$ & $1.38(.60-3.17)$ \\
\hline \multicolumn{5}{|l|}{ ESCOLARIDADE DA MÃE } \\
\hline$<4$ anos * & 33.9 & 6.5 & 29.8 & 4.8 \\
\hline 4 a 10 anos & 33.2 & 7.0 & 36.2 & 2.9 \\
\hline 11 anos & 36.3 & 3.3 & 46.8 & 5.1 \\
\hline
\end{tabular}

\begin{tabular}{lcccc}
\hline RP (IC 95\%) & $.98(.76-1.27)$ & $1.07(.54-2.13)$ & $1.22(.93-1.59)$ & $.61(.25-1.52)$ \\
\hline & $1.07(.84-1.37)$ & $.51(.23-1.11)$ & $1.57(1.22-2.03)$ & $1.07(.48-2.39)$ \\
\hline CLASSE ECONôMICA & \multicolumn{5}{l}{} \\
\hline A e B * & 40.4 & 3.7 & 53.4 & 5.0 \\
\hline C e D & 33.5 & 5.7 & 36.6 & 4.0 \\
\hline RP (IC 95\%) & $.83(.67-1.03)$ & $1.52(.66-3.52)$ & $.69(.58-81)$ & $.82(.38-1.74)$ \\
\hline
\end{tabular}

*Categoria de referência. As variáveis cor de pele, estado civil, campus, turno e período

apresentaram valor-p > 20 e não foram inseridas no quadro. RP: razão de prevalência.

No quadro 4 estão apresentados os resultados dos modelos múltiplos que identificaram as variáveis associadas aos comportamentos de risco investigados. Com relação ao gênero, os homens apresentaram maior prevalência em envolvimento em brigas $(R P=2.04)$.

Os estudantes com idade até 21 anos apresentaram maior prevalência para o não uso de cinto de segurança $(R P=1.32)$ e não uso de capacete $(R P=1.87)$, ao serem comparados com aqueles com idade superior a 21 anos.

Em relação à área de graduação, os estudantes da área de ciências exatas apresentaram menor prevalência para o não uso de cinto de segurança ( $R P=.65)$ e bebida alcoólica associada à direção de veículo ( $R P=.64)$, quando comparados com os da área das ciências biológicas e da saúde.

Os estudantes que não trabalham apresentaram prevalência menor em bebida alcoólica associada à direção de veículo ( $\mathrm{RP}=.73$ ), quando comparados com aqueles que trabalham com vínculo.

Com relação à escolaridade, os estudantes, cuja escolaridade do pai é maior, apresentaram uma prevalência menor ( $\mathrm{RP}=.24)$, em não uso de capacete, enquanto que aqueles, cuja escolaridade da mãe é maior, apresentaram uma prevalência maior em bebida alcoólica associada à direção de veículo.

Os estudantes pertencentes à classe econômica $\mathrm{C}$ e $\mathrm{D}$ mostraram uma prevalência menor em bebida alcoólica associada a veículo ( $\mathrm{RP}=.77$ ).

QUADRO 4. Razão de prevalência (RP) ajustada para comportamentos relacionados à segurança pessoal entre universitários no norte de Minas Gerais, Brasil, 2013

\begin{tabular}{|c|c|c|c|c|}
\hline \multirow[t]{2}{*}{ VARIÁVEIS } & $\begin{array}{l}\text { NÃO USO DE CINTO } \\
\text { DE SEGURANÇA }\end{array}$ & $\begin{array}{c}\text { NÃO USO } \\
\text { DE CAPACETE }\end{array}$ & $\begin{array}{l}\text { BEBIDA ALCOÓLICA } \\
\text { ASSOCIADA } \\
\text { A DIREĈAOO } \\
\text { DE VEÍCULO }\end{array}$ & $\begin{array}{l}\text { ENVOLVIMENTO } \\
\text { EM BRIGAS }\end{array}$ \\
\hline & RP(IC 95\%) & RP(IC 95\%) & RP(IC 95\%) & RP(IC 95\%) \\
\hline \multicolumn{5}{|l|}{ GÊNERO } \\
\hline Feminino * & & & & 1.00 \\
\hline Masculino & n.s. & n.s. & n.s. & $2.04(1.10-3.79)$ \\
\hline
\end{tabular}




\begin{tabular}{|c|c|c|c|c|}
\hline Acima de 21 anos * & 1.00 & 1.00 & n.s. & n.s. \\
\hline Até 21 anos & $1.32(1.09-1.159)$ & $1.87(1.04-3.37)$ & & \\
\hline \multicolumn{5}{|l|}{ ÁREA DA GRADUAÇão } \\
\hline Biológicas e da Saúde * & 1.00 & & 1.00 & \\
\hline Humanas & $1.07(.85-1.35)$ & n.s. & $.73(.59-.90)$ & n.s. \\
\hline Exatas e Tecnológicas & $.65((.17-.91)$ & & $.64(.48-.85)$ & \\
\hline Sociais Aplicadas & $.80(.62-1.04)$ & & $.76(.62-.94)$ & \\
\hline \multicolumn{5}{|l|}{ TRABALHO } \\
\hline Trabalha com vínculo* & & & 1.00 & \\
\hline Trabalha sem vínculo & n.s. & n.s. & .77(.59-1.01) & n.s. \\
\hline Não trabalha & & & $.73(.61-.87)$ & \\
\hline \multicolumn{5}{|l|}{ ESCOLARIDADE DO PAI } \\
\hline$<4$ anos* & & 1.00 & & \\
\hline 4 a 10 anos & n.s. & $.61(.34-1.10)$ & n.s. & n.s. \\
\hline 11 anos & & $.24(.10-.57)$ & & \\
\hline \multicolumn{5}{|l|}{ ESCOLARIDADE DA MÃE } \\
\hline$<4$ anos * & & & 1.00 & \\
\hline 4 a 10 anos & n.s. & n.s. & $1.13(0.86-1.48)$ & n.s. \\
\hline 11 anos & & & $1.43(1.10-1.86)$ & \\
\hline \multicolumn{5}{|l|}{ CLASSE ECONÔMICA } \\
\hline $\mathrm{AeB}^{*}$ & & & 1.00 & \\
\hline CeD & n.s. & n.s. & $.77(.64-.91)$ & n.s. \\
\hline
\end{tabular}

n.s.: não significativo * categoria de referência

\section{DISCUSSÃO}

O presente estudo avaliou os comportamentos de risco para a saúde associados à segurança pessoal em uma população de universitários, onde observou-se que esses jovens estão cada vez mais expostos a atitudes de risco. Essas situações podem levar a um aumento da incidência de acidentes e violências, gerando altos custos emocionais, sociais e gastos com a saúde, podendo comprometer a sua vida e a de outros (Mascarenhas et al., 2009).

Os comportamentos mais prevalentes foram: não uso de cinto de segurança e bebida associada à direção de veículo, sendo também mais prevalentes entre os homens e os mais jovens. Prevalências menores foram encontradas em estudos prévios, entre aqueles que não usavam cinto de segurança (Faria, Gandolfi, \& Moura, 2014; Malta et al., 2016; Neto, et al., 2010). Quanto à bebida associada à direção de veículo, prevalências menores também foram encontradas em inquéritos da Vigilância de Fatores de Risco e Proteção para Doenças Crônicas por Inquérito Telefônico (VIGITEL) e Pesquisa Nacional de Saúde (PNS) realizados nas capitais brasileiras e no Distrito Federal. Esses estudos também mostraram

prevalências mais elevadas para os homens e para os mais jovens corroborando os resultados do presente estudo (Malta et al., 2015).

Mais da metade dos jovens apresentaram simultaneamente os comportamentos de risco mais prevalentes, uso de bebida alcoólica associada à direção de veículo e não uso de cinto de segurança. Para alguns jovens, a entrada para a universidade, representa vivenciar novas experiências, inclusive, a maior participação em festas com bebidas, podendo levar a condutas inesperadas como, dirigir embriagado (Chavez, O'Brien, \& Pillon, 2005; Peuker, Fogaça, \& Bizarro, 2006)

$\mathrm{Na}$ análise múltipla observou-se que e os homens possuem mais chances de envolvimento com brigas. Resultado semelhante foi encontrado em um estudo prévio, onde observou-se que adolescentes do gênero masculino, têm maior probabilidade de se envolver em briga (Silva et al., 2009). Resultados significativos para o gênero masculino também foram encontrados em outros estudos prévios (Bernadelli Junior, 2010; Castro, Cunha, \& Souza, 2011; Farias et al., 2009; Malta et al., 2010). Esse achado reflete as questões de gênero em que o sexo masculino associa-se à vivência e expressão da agressividade e às normas sociais e culturais da nossa sociedade, onde brigar é um comportamento relativamente aceitável entre os homens (Malta et al., 2010; Silva, et al., 2009).

Os resultados também revelaram que os mais jovens possuem maior chance de não usar o cinto de segurança e não usar capacete. 0 não uso desses equipamentos de proteção amplia as chances de acidentes graves, mortes e incapacidades, mas quando usado corretamente reduz o número lesões traumáticas graves (Neto et al., 2010; Silva, Barbosa, $\&$ Chavaglia, 2010). Apesar de haver um aumento do uso desses equipamentos, o uso do cinto no banco de trás, ainda é pouco usado no país. Ações devem ser desenvolvidas para reduzir as lesões e mortes no trânsito (Malta et al., 2016)

Com relação à área de graduação, os estudantes da área de ciências exatas apresentaram resultado significativo para não uso de cinto de segurança e os acadêmicos das áreas de humanas, exatas e sociais aplicadas mostraram resultado significativo para o consumo de bebida alcoólica associada à direção de veículo, apresentando menor prevalência desse comportamento, quando comparados à área de ciências biológicas e da saúde.

oi também encontrado resultado significativo para quem não trabalha, apresentando menor prevalência do uso de bebida alcoólica associada à direção de veículo. Provavelmente esse achado seja explicado pelo fato de que, quem não exerce atividades trabalhistas tem menor exposição ao trânsito diariamente (Magalhães, Lopes, Koifman, \& Muniz, 2011), como também o poder aquisitivo pode dificultar o acesso à bebida alcoólica, bem como o acesso a veículos.

A escolaridade alta do pai apresentou associação significativa com menor prevalência do não uso de capacete. A escolaridade alta da mãe mostrou resultado significativo para maior prevalência de uso de bebida alcoólica associada à direção de veículo. Resultado semelhante foi encontrado no estudo realizado com escolares nas capitais brasileiras, em que a escolaridade alta da mãe foi associada ao uso de bebida e direção (Malta, Mascare- 
nhas, Porto, Barreto, \& Neto, 2014). Talvez isso se deve ao fato de que, a mulher com maior escolaridade está ocupando cada vez mais o mercado de trabalho e, por trabalhar o dia inteiro, não tem tempo disponível para assistência aos filhos, ficando longe da vida cotidiana deles, deixando-os mais vulneráveis a situações de riscos (Assis, 2009).

Quanto à classe econômica $C$ e D, o resultado foi significativo para bebida associada a veículo, mostrando comportamento de risco menor em comparação com a classe A e B. A classe social elevada pode favorecer a aquisição de veículos e um maior consumo de bebidas. Um melhor nível socioeconômico amplia o acesso ao veículo motorizado (Duarte, \& Garcia, 2014). Existem algumas limitações em nosso estudo, como o delineamento transversal, por inviabilizar relações de causalidade e a utilização de questionários autorreferidos para determinação dos CRS, sendo possível que tais prevalências tenham sido subestimadas.

\section{CONCLUSÃo}

Os CRS relacionados à segurança pessoal, que apresentaram as maiores prevalências foram: não uso de cinto de segurança e bebida alcoólica associada à direção de veículo, sendo que mais da metade dos jovens apresentou simultaneamente os comportamentos de risco mais prevalentes. Os mais jovens se mostraram em situações de risco em não fazer uso de cinto de segurança e de capacete, assim como os jovens do sexo masculino, se envolvendo em brigas. Este estudo mostrou que os universitários, apesar de possuírem um nível de instrução mais elevado, estão se arriscando no trânsito e colocando suas vidas e de outros em perigo. Sugere-se que sejam realizadas medidas educativas voltadas para esses jovens e suas famílias, direcionadas à conscientização e sensibilização para a condução segura no trânsito, iniciadas nos primeiros anos escolares.
Associação Brasileira de Empresas de Pesquisa. (2013). Critério de classificação econômica Brasil: Dados con base no levantamento sócio econômico 2000. Disponivel em http://www.abep.org/criterio-brasil

Assis, R. H. (2009). A Inserção da mulher no mercado de trabalho. In VI Congresso Virtual Brasileiro de Administração. Disponivel em http://www.convibra. org/2009/artigos/140_0.pdf

Barbosa, F. N. M. (2014). Comportamento de risco à saúde de adolescentes. Dissertação de mestrado, Universidade Estadual do Sudoeste da Bahia, Brasil Dispersidade Estadul do Sudoeste da Bahia, Brasil. Disponivel em http.//Www.uesb.br/ppgenfsaude/dissertaBernadeli Junior, R. (2010). Comportamentos de risco para a saúde de estudantes da Universidade Estadual do Norte do Paraná, Brasil. Tese de doutorado, Universidade de Trás-os-Montes e Alto Douro, Vila Real, Portugal. Disponível em https://xa.yimg.com/kg/groups/21808523/... tese+revisão+final+nuno+-+pronta.doc

Castro, M. L., Cunha, S. S., \& Souza, D. P. O. (2011). Comportamento de violência e fatores associados entre estudantes de Barra do Garças, MT. Revista de Saúde Pública, 45(6), 1054-1061. doi:10.1590/s003 89102011005000072

Centers for Disease Control and Prevention (2016) Youth risk behavior surveillance - United States, 2015. MMWR Surveillance Summaries, 65(6). Disponivel em ss6506_updated.pdf Chavez, K. A., O'Brien, B., \& Pillon, S.C. (2005). Uso de drogas e comportamentos de risco no contexto de uma comunidade universitária. Revista Latino-Americana Enfermagem, 13(2), 1194-1200. doi:10.1590/ S0104-11692005000800014

Duarte, E. C., \& Garcia, L. P. (2014). Motoristas adolescentes no Brasil: Prevalência e fatores associados estimados a partir da Pesquisa Nacional de Saúde do Escolar (PeNSE 2012). Revista Brasileira de Epidemiologia 17(Supl.1). do:10.1590/1809-4503201400050002 Faria, Y. O., Gandolfi, L., \& Moura, L. B. A. (2014). Prevalência de comportamentos de risco em adulto jovem e universitário. Acta Paulista de Enfermagem, 27(6) 591-595. doi:10.1590/1982-0194201400096

Farias Junior, J. C., Nahas, M. V., Barros, M. V. G., Loch, M. R., Oliveira, E. S. A., de Bem, M. F. L., \& Lopes, A. S. (2009). Comportamentos de risco à saúde em adolescentes no sul do Brasil: Prevalência e fatores associados. Revista Panamericana de Salud Publica, 25(4) 344-52. doi:10.1590/s1020-49892009000400009
Guedes, D. P., \& Lopes, C. C. (2010). Validação da versão brasileira do Youth Risk Behavior Survey 2007. Revista de Saúde Pública, 44(5), 840-850. doi:101590/ s0034-89102010000500009

Kanchan, T., Kulkarni, V., Bakkannavar, S. M., Kumar, N. \& Unnikrishnan, B. (2012). Analysis of fatal road traffic accidents in a coastal township of South India. The Journal of Forensic and Legal Medicine. 19, 448451. doi:10.1016/j.jflm.2012.02.031

Magalhães, A. F., Lopes, C. M., Koifman, R. J., \& Muniz, P. T. (2011). Prevalência de acidentes de trânsito autorreferidos em Rio Branco, Acre. Revista de Saúde Pública, 45(4), 738-744. doi:10.1590/S003489102011005000031

Malta, D. C., Andrade, S. S. C. A., Gomes, N., Silva, M. M. A., Neto, O. L. M., Reis, A. A. C., \& NardI, A. C. F. (2016). Lesões no trânsito e uso de equipamento de proteção na população brasileira, segundo estudo de base podoi:10.1590/1413-81232015212.23742015

Malta, D. C., Bernal, R. T. I., Mascarenhas, M. D. M. Silva, M. M. A., Szwarcwald, C. L., \& Neto, O. L. M. (2015). Consumo de bebidas alcoólicas e direção de veículos nas capitais brasileiras e no Distrito Federal, segundo dois inquéritos nacionais de saúde. Revista Brasileira Epidemiololgia, 18(Supl 2), 214-223. doi:10.1590/1980-5497201500060019

Malta, D. C., Mascarenhas, M. D. M. M., Porto, D. L. Barreto, S. M., \& Neto, O. L. M. (2014). Exposição ao álcool entre escolares e fatores associados. Revista de Saúde Pública, 48(1), 52-62. doi:10.1590/500348910.2014048004563

Malta, D. C., Souza, E. R., Silva, M. M. A., Silva, C. S. Andreazzi, M. A. R., Crespoi, C., Mascarenhas, M. D. M. M. Penna G O (2010) Vivência de violência entre escolares brasiteros: resultados da Pesquisa Nacional de Saúde do Escolar (PeNSE). Ciência \& Saúde Coletiva, 15(Supl 2), 3053-3063. doi:10.1590/S141381232010000800010

Mascarenhas, M. D. M., Malta, D. C., Silva, M. M. A., Carvalho, C. G., Monteiro, R. A., \& Neto, O. L. M. (2009). Consumo de álcool entre vitimas de acidentes e violências atendidas em serviços de emergência no Brasil, 2006 e 2007 Ciência \& Saúde Coletiva, 14(5), 1780-1796. doi:10.1590/S1413-81232009000500020

Ministério da Saúde (2015). Brasil reduz em 5,7\% número de mortes no trânsito. Disponível em http:// www.brasil.gov.br/saude/2015/11/brasil-reduz-em-5-7-numero-de-mortes-no-transito 
Neto, O. L. M., Malta, D. C., Mascarenhas, M. D. M., Duarte, E. C., Silva, M. M. A., Oliveira, K. B.,... Porto, D. L. (2010). Fatores de risco para acidentes de transporte terrestre entre adolescentes no Brasil: Pesquisa $\mathrm{Na}$ cional de Saúde do Escolar (PeNSE). Ciência \& Saúde Coletiva, 15(Supl. 2), 3043-3052. doi:10.1590/S141381232010000800009

Organização das Nações Unidas. (2015). ONU: Acidentes no trânsito ainda matam 1,25 milhão por ano, 90\% em países de renda média e baixa. Disponivel em https://nacoesunidas.org/onu-acidentes-no-transito-ainda-matam-125-milhao-por-ano-90-em-paises-de-renda-media-e-baixa/

Ministérios da Sáde. (2015). Pesquisa Nacional de Saúde 2013: Acesso e utilização dos serviços de saúde, acidentes e violências. Brasil, grandes regiões e unidades da federação. Rio de Janeiro: Instituto Brasileiro de Geografia e Estatística, Coordenação de Trabalho e Rendimento. Disponivel em biblioteca.ibge.gov.br/ visualizacao/livros/liv94074.pdf

Peuker, A. C., Fogaça, J., \& Bizarro, J. L. (2006). Expectativas e beber problemático entre pectativas e be rios. Psicologian. Teoria e Pesquisa, 22(2).

Silva, D. P., Barbosa, M. H., \& Chavaglia, S. R. R. (2010) Utilização de equipamentos de segurança entre vítimas de acidentes no município de Uberaba-MG. Revista Eletrônica de Enfermagem,12(1), 83-88. doi:10.5216/ree.v12i1.5482.

Silva, R. A., Jansen, K., Godoy, R. V., Souza, L. D. M., Horta, B. L., \& Pinheiro, R. T. (2009). Prevalência e fatores associados a porte de arma e envolvimento em agressão física entre adolescentes de 15 a 18 anos: Estudo de base populacional. Cadernos de Saúde Pública, 25(12), 2737-2745. doi:10.1590/s0102-311×2009001200020
AUTORES:

Ivanete Fernandes do Prado ${ }^{1,2}$

Berta Leni Costa Cardoso ${ }^{1}$

Elionara Teixeira Boa Sorte ${ }^{1}$

Marcela Andrade Rios

Nanci Maria de França ${ }^{2}$

Universidade do Estado da Bahie UNEB, Brasil

Universidade Católica de Brasília,

UCB, Brasil

https://doi.org/10.5628/rpcd.17 S3A.65
Hipertensão arterial

\section{durante a gravidez}

\section{PALAVRAS CHAVE:}

Síndromes hipertensivas.

Prematuridade. Baixo peso ao nascer.

Promoção de saúde. Exercício físico.

\section{RESUMO}

Este estudo objetivou identificar a prevalência de gestantes com a hipertensão arterial durante a internação para o parto e associá-la com a prematuridade e o baixo peso ao nascer. Trata-se de estudo com delineamento tipo caso controle no qual as associações das variáveis preditoras com desfecho prematuridade e baixo peso ao nascer foram analisadas por meio de modelo hierarquizado de regressão logística múltipla. A prevalência de gestantes com hipertensão arterial durante a internação para o parto na amostra estudada foi de $13.5 \%$. Foram observadas associações significativas da hipertensão arterial tanto com a prematuridade, quanto com o baixo peso ao nascer. Gestantes com hipertensão apresentam 3.47 (IC95\%: 1.37 - 8.81) vezes mais chance de ter filho prematuro e 2.55 (1.03 6.32) vezes mais chance de ter filho com baixo peso do que aquelas sem fatores de risco à gestação. Gestantes hipertensas devem ser encaminhadas e asseguradas no serviço de acompanhamento de alto risco, bem como orientadas sobre a prática de exercícios físicos com o intuito de diminuir as complicações perinatais e maternas. 